\title{
Investigation on Buckling of Orthotropic Circular and Annular Plates of Continuously Variable Thickness by Optimized Ritz Method
}

\author{
Fatemeh Farhatnia $^{1, \mathrm{a}}$, Arash Golshah ${ }^{2}$ \\ ${ }^{1}$ Islamic Azad University-Branch of Khomeinishahr,Mechanical Engineering Faculty, Boulvard Manzarie, Khomeinishahr, Isfahan, Iran \\ ${ }^{2}$ Iran Aircraft Manufacturing (HESA), Moallem Highway, Isfahan, Iran
}

Received 10 May 2010, Accepted 15 September 2010

\begin{abstract}
This paper investigates symmetrical buckling of orthotropic circular and annular plates of continuous variable thickness. Uniform compression loading is applied at the plate outer boundary. Thickness varies linearly along radial direction. Inner edge is free, while outer edge has different boundary conditions: clamped, simply and elastically restraint against rotation. The optimized Ritz method is applied for buckling analysis. In this method, a polynomial function that is based on static deformation of orthotropic circular plates in bending is used. Also, by employing an exponential parameter in deformation function, eigenvalue is minimized in respect to this parameter. The obtained results show that in plate with identical thickness, increasing of outer radius decreases the buckling load factor
\end{abstract}

Keywords: Buckling, Orthotropic circular and annular plates, Optimized Ritz, Variable thickness.

\section{Introduction}

Orthotropic circular and annular plates are always used by mechanical, civil, aerospace and structural engineers and designers. Some applications of these systems are pressure vessel valve, reinforced circular plates by radial and circumference supporter, composite plates, cylinder head cover, bulkhead plates in submarines, separated plates in aircraft, optical lenses, and acoustic transducers in rockets. For first time Woinosky[1], studied the problem of elastic stability of orthotropic circular plates. He introduced numeric results by using Bessel function for buckling of plates. Menk et al.[2], studied on variation of thickness on buckling of orthotropic rectangular plate. Laura et al. [3], found critical load of buckling for isotropic annular plate with constant thickness by optimized Ritz method. Imposed boundary condition of plate for inner edge either outer edge was under different supports. Cianco [4] studied on buckling of circular and annular isotropic plate with variable thickness that used as a part of submarine. This plate was considered with free support on inner edge and clamped support and resistant of rotation for outer edge. The thickness of plate is exponential function of its radius. He analyzed the plate by optimized Ritz method. Bremec et al. [5] also introduced one optimized rate of variation of thickness for buckling of isotropic plates which both in inner edge and outer edge was under constant radial load and thickness was varied in radial direction. Buckling function was in linear fashion and solved by numeric method under simply and clamped supported. Coutierrez et al. [6] considered buckling and vibration of the isotropic plate with variable thickness on elastic support using Ritz method, and obtained acceptable results. Liang et al. [7] found natural frequencies of one orthotropic circular and annular plate with variable thickness using Ritz method and compared with the result of finite element method that were good in agreement between them.

In this paper, buckling of orthotropic circular and annular plates with linear variation of thickness under constant compressive radial loading is studied. The boundary condition of annular plates are F-C plates (free inner, and clamped outer edge) or clamped circular solid ones, F-S plates (free inner, simply outer edge) or simply supported solid circular ones, and annular plates with free inner support and resistant elastic against rotation outer edge. Circular plates contain plates with clamped, simply and elastic resistant against rotation boundary condition. Solving buckling differential equation of orthotropic circular or annular plate with variable thickness is impossible by analytical method and it should be solved using numerical or energy method. For this reason optimized Ritz method is used. The results of this method is more precisely than Ritz method. For optimization of Ritz method, one exponential parameter in approximate function is considered eigenvalues (buckling load factor) that is obtained, are minimized according to this exponential parameter. Furthermore, the comparison between results of this method and the results of finite element procedure is done. The effects of thickness variation, boundary conditions, young module ratio in radius and circumference axis, variation of ratio of inner radius to outer one on buckling load factor are considered.

\section{Theory}

\subsection{Basic formulation of the problem}

The formulation of the problem is derived under the following assumptions:

1. The plate is in the state of plane stress.

2. The stress-strain relationship follows of orthotropic material.

${ }^{\mathrm{a}}$ Corresponding author: farhatnia@iaukhsh.ac.ir 
3. The plate is thin, therefore the Kirchhoff assumptions incorporated.

4. The thickness is varied in the direction of radius of the plate.

Consider a circular annular plate with variable thickness $\mathrm{h}(\mathrm{r})$, $\mathrm{a}, \mathrm{b}$ inner and outer radius, respectively as shown in Figure (1). For buckling analysis, the in-plane displacement $\mathrm{u}$ and $\mathrm{v}$ may be neglected and only out-of-plane deformation $\mathrm{w}$ is considered.

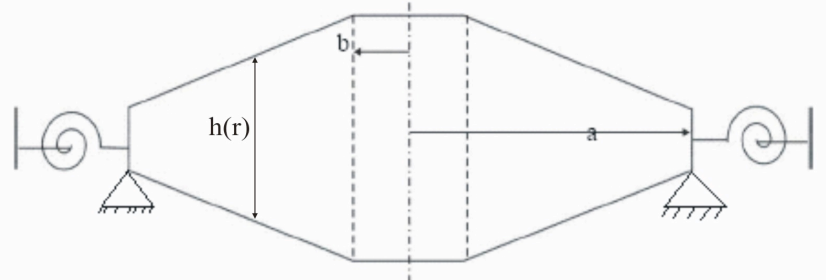

Fig. 1. Schematic view of annular plate with variable thickness

The governing energy functional can be given by:

$$
J=U+V
$$

Where $U$ is the stored strain energy per unit volume that in the polar coordinate system for plane stress is given as follows [8]:

$$
U=\frac{1}{2} \iint_{v}\left(\oint_{r} \varepsilon_{r}+\sigma_{\theta} \varepsilon_{\theta}+\tau_{r \theta} \gamma_{r \theta}\right) r d r d \theta d z
$$

The work done by in-plane radial force is given as:

$$
V=\frac{1}{2} \iint_{A}\left[N_{r}\left(\frac{\partial w}{\partial r}\right)^{2}+N_{\theta}\left(\frac{\partial w}{\partial \theta}\right)^{2}\right] r d r d \theta
$$

In present study, the axial radial symmetry is assumed, so plate is independent of azimuthal variable:

$$
V=\pi \int_{b}^{a} N_{r}\left(\frac{\partial w}{\partial r}\right)^{2} r d r
$$

For orthotropic annular plate, $\mathrm{V}$ is derived as follows [9]:

$$
V=\pi \int_{b}^{a} \frac{-N_{0}}{h_{a}} \frac{a^{\beta+1}}{a^{2 \beta}-b^{2 \beta}}\left[r^{\beta-1}-\frac{b^{2 \beta}}{r^{\beta+1}}\right]\left(\frac{\partial w}{\partial r}\right)^{2} r d r
$$

Also, using the stress-strain relation for orthotropic material, substituting into equation (1), one may obtain:

$$
U=\frac{1}{2} \iint \frac{z^{2}}{\int-v_{r} v_{\theta}}\left[E_{r}\left(\frac{d^{2} w}{d r^{2}}\right)^{2}+2 E_{r} v_{\theta}\left(\frac{d^{2} w}{d r^{2}}\right)\left(\frac{d w}{d r}\right)+E_{\theta}\left(\frac{d w}{r d r}\right)^{2}\right]
$$

where,

$$
D_{r}(r)=\frac{E_{r} h^{3}(r)}{12\left(1-v_{r} v_{\theta}\right)} \quad, \quad \frac{E_{\theta}}{E_{r}}=\frac{D_{\theta}(r)}{D_{r}(r)}=\beta^{2}
$$

In above equation, $D_{r}(r), D_{\theta}(r)$ denote circumferential and radial bending stiffness of the plate, respectively.

In this study, annular and solid circular plates with continuously varying thickness are considered. The variation of thickness along the radius direction can be expressed as follows:

$$
h(r)=h_{o}\left(1+\gamma\left(\frac{r}{a}\right)^{n}\right)
$$

where ho and $a$ represent the thickness in the centre (inner radius for annular plate) and the outer radius of plate, respectively.
In order to consider the influence of non uniform thickness on buckling load factor, two values are assigned for the parameter $\mathrm{n}, \mathrm{n}=0,1$ for uniform and linearly varying thickness, respectively. $\gamma$ is non-dimensional geometric parameter that may be positive (centrally thinner circular plate ) or negative (centrally thicker circular plate) and is defined as follows:

For circular solid plates:

$$
\gamma=\frac{h_{a}}{h_{o}}-1
$$

For circular annular plates:

$$
\gamma=\frac{h_{b}-h_{o}}{h_{o}\left(r_{b}\right)^{n}}
$$

In above equation, $\mathrm{r}_{\mathrm{b}}$ is the ratio of inner radius to the outer one $\left(=\frac{b}{a}\right)$.

In the sake of convenience, the variables in equations (7) and (15) are transformed to dimensionless one, so the total potential energy functional is as follows:

$$
\begin{aligned}
\frac{a^{2}}{\pi D_{0}} J(w)= & \int_{r_{b}}^{1}\left\{g(R)\left[\left(\frac{d^{2} w}{d R^{2}}\right)^{2}+2 v_{\theta}\left(\frac{d^{2} w}{d R^{2}}\right)\left(\frac{d w}{R d R}\right)+\beta^{2}\left(\frac{d w}{R d R}\right)^{2}\right]\right. \\
& \left.-\frac{\lambda}{1-r_{b}^{2 \beta}}\left[R^{\beta-1}-\frac{r_{b}^{2 \beta}}{R^{\beta+1}}\right]\left(\frac{d w}{d R}\right)^{2}\right\} R d R
\end{aligned}
$$

that,

$$
g(R)=(1+\gamma R)^{3}, \quad D_{0}=\frac{E_{r} h_{o}^{3}}{12\left(1-v_{r} v_{\theta}\right)}, \quad R=\frac{r}{a}
$$

$\lambda$ is the buckling load factor that is related to buckling load as follows:

$$
\lambda=\frac{N_{0} a^{2}}{D_{0}}
$$

\subsection{Optimized Ritz method}

Regarding to this fact that Ritz method is an upper bound method, so determined eigenvalue is more than real one, therefore if one can optimized it somehow, the results will be closer to real one. In general, if the function which introducing the unknown quantity, is a linear combination of shape modes $\varphi_{n}$, as follows:

$$
f(x)=\sum_{n=1}^{N} c_{n} \varphi_{n}(x)
$$

where $c_{n}$ is the unknown constants. Regarding to the idea of optimization, by performing the optimized Ritz method, it is quite convenient to approximate the displacement amplitude $\mathrm{W}(\mathrm{R})$ by means of a summation [4]:

$$
f(x)=\sum_{n=1}^{N} c_{n} \varphi_{n}(x, k)
$$

Note that $k$ in above equation is the exponential optimization parameter. Regarding to the equation (15), out-plane displacement is given as follows:

$$
w(R)=\sum_{i=1}^{N} c_{i} w_{i}(R, k)
$$

By minimizing potential energy functional in Ritz method: 


$$
\frac{\partial J}{\partial c_{i}}=0 \quad i=1, \ldots, N
$$

Total number of $\mathrm{N}$ linear homogenous algebraic equations are generated that the unknowns are constants $\mathrm{c}_{\mathrm{n}}$. It forms the eigenvalue problem that the eigenvlaues are the values of buckling load parameter. The non-trivially condition leads to a transcendental equation in whose lowest root is the desired buckling load factor [4].

Since $\frac{\partial \lambda}{\partial k}=0$, by requiring one is able to optimize the fundamental eigenvalue.

\subsection{Buckling of annular circular plate}

Regarding to the equation $(15), w_{i}(R)$ is defined as follows [9]:

$$
w_{i}(R, k)=\left(a_{i} R^{k}+b_{i} R^{1+\beta}+1\right) k^{i-1}
$$

Unknown constants $a_{i}$ and $b_{i}$ are determined by applying boundary conditions.

\subsubsection{Clamped outer edge ( $F-C)$ plate}

For a clamped outer edge; in $\mathrm{r}=\mathrm{a}$ or $\mathrm{R}=1$, the governing boundary conditions are:

$$
\left\{\begin{array}{l}
w_{i}(1)=0 \\
\frac{d w_{i}}{d R}(1)=0
\end{array}\right.
$$

By substitution eq. (19) into eq. (20), the $a_{i}$ and $b_{i}$ values are determined as follows:

$$
a_{i}=\frac{-1-\beta}{1+\beta-k} \quad b_{i}=\frac{k}{1+\beta-k}
$$

\subsubsection{Simply supported outer edge(S-F) plate}

For a simply supported outer edge, the out-plane displacement $\mathrm{w}$, must be satisfied the following conditions:

$$
\left\{\begin{array}{l}
w(1)=0 \\
\frac{d^{2} w}{d R^{2}}(1)+v_{\theta} \frac{d w}{d R}(1)=0
\end{array}\right.
$$

Regarding to the boundary condition in outer edge, the $\mathrm{a}_{\mathrm{i}}$ and $b_{i}$ values are determined:

$$
\begin{aligned}
& a_{i}=\frac{(1+\beta)\left(-2+2 i+\beta+v_{\theta}\right)}{(-1-\beta+k)\left(-2+2 i+k+\beta+v_{\theta}\right)} \\
& b_{i}=-\frac{k\left(-3+2 i+k+v_{\theta}\right)}{(-1-\beta+k)\left(-2+2 i+k+\beta+v_{\theta}\right)}
\end{aligned}
$$

\subsubsection{Elastically restrained rotation}

For the case of elastically restrained rotation:

$$
\left\{\begin{array}{l}
w_{i}(1)=0 \\
\varphi \frac{d w_{i}}{d R}(1)=-g(R)\left[\frac{d^{2} w_{i}}{d R^{2}}(1)+v_{\theta} \frac{d w_{i}}{d R}(1)\right]
\end{array}\right.
$$

the $a_{i}$ and $b_{i}$ values are determined as follows:

$$
a_{i}=-\frac{Q_{i}-L_{i}}{S_{i}-L_{i}}, \quad b_{i}=-\frac{S_{i}-Q_{i}}{S_{i}-L_{i}}
$$

In above equation, $S_{i}, Q_{i}, L_{i}$ are defined as follows:

$$
\begin{aligned}
S_{i} & =(k+i-1)\left[1+g(R) \varphi\left(-2+i+k+v_{\theta}\right)\right] \\
L_{i} & =(i+\beta)\left[1+g(R) \varphi\left(-1+i+\beta+v_{\theta}\right)\right] \\
Q_{i} & =(i-1)\left[1+g(R) \varphi\left(-2+i+v_{\theta}\right)\right]
\end{aligned}
$$

$$
\varphi=\frac{a k_{\varphi}}{D_{0}} \text { represents the dimensionless flexibility co- }
$$
efficient. Using the equation (18), total number of $\mathrm{N}$ linear homogenous algebraic equations is generated. The nontrivially the determinant $|A-\lambda B|=0$ of coefficients matrix, as follows:

Where $\mathrm{A}_{\mathrm{ij}}$ and $\mathrm{B}_{\mathrm{ij}}$ are determined as follows:

$$
\begin{array}{r}
A_{i j}=\int_{r_{b}}^{1} g(r)\left[\left(\frac{d^{2} w_{i}}{d R^{2}}\right)\left(\frac{d^{2} w_{j}}{d R^{2}}\right)+2 v_{\theta}\left(\frac{d^{2} w_{i}}{d R^{2}}\right)\left(\frac{d w_{j}}{R d R}\right)\right. \\
\left.+\beta^{2}\left(\frac{d w_{i}}{R d R}\right)\left(\frac{d w_{j}}{R d R}\right)\right] R d R+\varphi\left(\frac{d w_{i}}{d R}\right)\left(\frac{d w_{j}}{d R}\right) \\
B_{i j}=\frac{\lambda}{1-r_{b}^{2 \beta}}\left[R^{\beta-1}-\frac{r_{b}^{2 \beta}}{R^{\beta+1}}\right]_{r b}^{1} \int\left(\frac{d w_{i}}{d R}\right)\left(\frac{d w_{j}}{d R}\right) R d R
\end{array}
$$

In the above equation, for the case of simply supported and clamped in outer edge, $\varphi=0, \varphi=\infty$ respectively.

\subsection{Buckling of circular solid plate}

Regarding to equation (17), for circular solid plate $\mathrm{w}_{\mathrm{i}}(\mathrm{R})$ is defined as follows [9]:

$$
w_{i}(R, k)=\left(a_{i} R^{k}+b_{i} R^{1+\beta}+1\right) k^{2(i-1)}
$$


In order to obtain $a_{i}$ and $b_{i}$ as constants, the following boundary conditions must be satisfied:

\subsubsection{Clamped outer edge, (C-F) plate}

$$
b_{i}=\frac{k}{1+\beta-k}, a_{i}=\frac{-1-\beta}{1+\beta-k}
$$

\subsubsection{Simply supported outer edge, (S-F) plate}

$$
\begin{aligned}
& a_{i}=\frac{(1+\beta)\left(-4+4 i+\beta+v_{\theta}\right)}{(-1-\beta+k)\left(-4+4 i+k+\beta+v_{\theta}\right)} \\
& b_{i}=-\frac{k\left(-5+4 i+k+v_{\theta}\right)}{(-1-\beta+k)\left(-4+4 i+k+\beta+v_{\theta}\right)}
\end{aligned}
$$

\subsubsection{Elastically restrained rotation}

$$
a_{i}=-\frac{Q_{i}-L_{i}}{S_{i}-L_{i}} \quad b_{i}=-\frac{S_{i}-Q_{i}}{S_{i}-L_{i}}
$$

\section{where,}

$$
\begin{aligned}
& S_{i}=(k+2 i-2)\left\lfloor 1+g(R) K_{\phi}\left(-3+2 i+k+v_{\theta}\right)\right\rfloor \\
& L_{i}=(2 i+\beta-1)\left[1+g(R) K_{\phi}\left(-2+2 i+\beta+v_{\theta}\right)\right] \\
& Q_{i}=2(i-1)\left[1+g(R) K_{\phi}\left(-3+2 i+v_{\theta}\right)\right]
\end{aligned}
$$

\section{$A_{i j}$ and $B_{i j}$ are determined as follows:}

$$
\begin{gathered}
A_{i j}=\int_{0}^{1} g(R)\left[\left(\frac{d^{2} w_{i}}{d R^{2}}\right)\left(\frac{d^{2} w_{j}}{d R^{2}}\right)+2 v_{\theta}\left(\frac{d^{2} w_{i}}{d R^{2}}\right)\left(\frac{d w_{j}}{R d R}\right)\right. \\
\left.+\beta^{2}\left(\frac{d w_{i}}{R d R}\right)\left(\frac{d w_{j}}{R d R}\right)\right]+\varphi\left(\frac{d w_{i}(1)}{R d R}\right)\left(\frac{d w_{j}(1)}{R d R}\right) \\
B_{i j}=\lambda R^{\beta-1} \int_{0}^{1}\left(\frac{d w_{i}}{d R}\right)\left(\frac{d w_{j}}{d R}\right) R d R
\end{gathered}
$$

Like before, in the above equation, for the case of simply supported $\varphi=0$ and clamped in outer edge, $\varphi=\infty$.

\section{Numerical results}

This section presents a number of numerical examples that shows the good performance of the proposed method, which was implemented in Mathematica 5.1 computer program. The results of the developed optimized Ritz method are

\begin{tabular}{|c|c|c|c|c|c|c|}
\hline \multirow{2}{*}{$\gamma$} & \multirow{2}{*}{$r_{b}$} & \multicolumn{5}{|c|}{$\mathbf{m}$} \\
\hline & & 1 & 2 & 3 & 4 & 5 \\
\hline \multirow{3}{*}{-0.3} & 0.1 & 8.06227 & 7.82111 & 7.80585 & 7.65965 & 7.65696 \\
\hline & 0.3 & 7.64248 & 7.26206 & 7.24417 & 7.15621 & 7.15612 \\
\hline & 0.5 & 10.7017 & 10.6339 & 10.6283 & 10.6069 & 10.6066 \\
\hline
\end{tabular}
compared with some other results obtained from FE method. All calculation has been performed for $\mathrm{E}_{\mathrm{r}}=10000 \mathrm{Mpa}, v_{\mathrm{e}}$ $=.3, \mathrm{a}=1 \mathrm{~m}, \mathrm{~h}_{\mathrm{o}}=.08 \mathrm{~m}$. It was revealed that convergent buckling load factor is obtained with 4-term series. Table (1) shows this convergence for centrally thicker annular orthotropic plate. In presenting results, the dimensionless buckling load factor is used. Value of this factor is obtained for plates of uniform and linearly continuously thickness.
Table (1): Convergence study for annular orthotropic plate

\section{Annular plate}

Table (2) depicts the influence of parameters $\gamma, \beta^{2}, r_{b}$ on the buckling load factor. It is observed with increasing the amount of $\beta$, buckling load factor is increased too. One observes that orthotropic plate $\left(\beta^{2}>1\right)$ has more stiffness against buckling occasion in comparison to isotropic one. Furthermore increasing parameter $\gamma$ toward positive values

\begin{tabular}{|c|c|c|c|c|c|c|}
\hline \multirow{2}{*}{$\beta^{2}$} & \multirow{2}{*}{$\gamma$} & \multicolumn{5}{|c|}{$r_{b}$} \\
\hline & & 0.1 & 0.2 & 0.3 & 0.4 & 0.5 \\
\hline \multirow{5}{*}{1} & -0.3 & 7.65965 & 7.08443 & 7.15621 & 8.17407 & 10.6069 \\
\hline & -0.1 & 11.6538 & 11.1526 & 11.9511 & 14.4785 & 19.7288 \\
\hline & 0 & 14.0102 & 13.6272 & 14.9707 & 18.5588 & 25.7602 \\
\hline & 0.1 & 16.605 & 16.4169 & 18.4444 & 23.3258 & 32.8914 \\
\hline & 0.3 & 23.5623 & 23.0035 & 26.8698 & 35.1226 & 50.8109 \\
\hline \multirow{5}{*}{2} & -0.3 & 12.2977 & 11.7989 & 11.3131 & 11.6165 & 13.4416 \\
\hline & -0.1 & 19.4974 & 18.8195 & 18.6013 & 20.0802 & 24.5068 \\
\hline & 0 & 23.8244 & 23.0811 & 23.1413 & 25.5038 & 31.7732 \\
\hline & 0.1 & 28.6657 & 27.8789 & 28.3333 & 31.807 & 40.3339 \\
\hline & 0.3 & 39.9593 & 39.1814 & 40.8333 & 47.3052 & 61.7521 \\
\hline \multirow{5}{*}{5} & -0.3 & 23.331 & 23.2335 & 22.7974 & 22.1416 & 22.4007 \\
\hline & -0.1 & 39.3318 & 39.151 & 38.4959 & 38.0138 & 39.9237 \\
\hline & 0 & 49.2861 & 49.0655 & 48.3052 & 48.0918 & 51.309 \\
\hline & 0.1 & 60.6435 & 60.3648 & 59.3648 & 59.7502 & 64.6481 \\
\hline & 0.3 & 87.8954 & 87.4584 & 86.6497 & 88.2489 & 97.7947 \\
\hline \multirow{5}{*}{10} & -0.3 & 39.4051 & 39.2595 & 39.2595 & 38.7452 & 37.9053 \\
\hline & -0.1 & 69.3379 & 69.0895 & 69.0894 & 68.2961 & 67.7093 \\
\hline & 0 & 88.3379 & 88.4204 & 88.1414 & 87.2232 & 87.0211 \\
\hline & 0.1 & 110.615 & 110.568 & 110.221 & 109.203 & 109.61 \\
\hline & 0.3 & 164.852 & 164.774 & 164.275 & 163.176 & 165.608 \\
\hline
\end{tabular}
makes the buckling load factor to increase. Regarding to table (1), some values of $\mathrm{r}_{\mathrm{b}}\left(\mathrm{r}_{\mathrm{b}}>\right.$.1) decreases the buckling load factor $\lambda$, meanwhile some other values makes it decrease. As shown in table (3), as it was expected, clamped boundary condition represents the highest value of factor while the simply supported one, shows the lowest. The influence of parameters $\gamma, \beta^{2}, r_{b}$ is the same as clamped case.

Table 2. Buckling load factor variation with simply supported outer edge 
F. Farhatnia et al: Investigation on buckling of orthotropic circular and annular plates of continuously variable thickness 131

Table 3. Buckling load factor variation with clamped outer edge

\begin{tabular}{|c|c|c|c|c|c|c|}
\hline \multirow{2}{*}{$\beta^{2}$} & \multirow{2}{*}{$\gamma$} & \multicolumn{5}{|c|}{$r_{b}$} \\
\hline & & 0.1 & 0.2 & 0.3 & 0.4 & 0.5 \\
\hline \multirow{5}{*}{1} & -0.3 & 2.38864 & 2.08242 & 1.74291 & 1.45192 & 1.22152 \\
\hline & -0.1 & 3.40413 & 2.99726 & 2.59067 & 2.25996 & 2.00307 \\
\hline & 0 & 3.99418 & 3.53995 & 3.10666 & 2.76324 & 2.50023 \\
\hline & 0.1 & 4.64426 & 4.14536 & 3.69047 & 3.33985 & 3.007638 \\
\hline & 0.3 & 6.13934 & 5.56135 & 5.8046 & 4.7343 & 4.48982 \\
\hline \multirow{5}{*}{2} & -0.3 & 3.865 & 3.70686 & 3.37779 & 2.95803 & 2.54578 \\
\hline & -0.1 & 5.91459 & 5.67527 & 5.2282 & 4.70576 & 4.21785 \\
\hline & 0 & 7.14892 & 6.86634 & 6.36338 & 5.79828 & 5.28355 \\
\hline & 0.1 & 8.53568 & 8.20873 & 7.65316 & 7.05245 & 6.51991 \\
\hline & 0.3 & 11.8043 & 11.3873 & 10.7398 & 10.0932 & 9.5504 \\
\hline \multirow{5}{*}{5} & -0.3 & 7.44953 & 7.42653 & 7.29997 & 6.95221 & 6.35169 \\
\hline & -0.1 & 12.4039 & 12.3628 & 12.1571 & 11.6415 & 10.8251 \\
\hline & 0 & 15.5186 & 15.467 & 15.2169 & 14.6124 & 13.6953 \\
\hline & 0.1 & 19.1024 & 19.0392 & 18.7421 & 18.0519 & 17.0371 \\
\hline & 0.3 & 27.8088 & 27.7192 & 27.3225 & 26.4616 & 25.2822 \\
\hline \multirow{5}{*}{10} & -0.3 & 12.7613 & 12.7592 & 12.7332 & 12.5904 & 12.1299 \\
\hline & -0.1 & 22.434 & 22.4299 & 22.3826 & 22.1401 & 21.4237 \\
\hline & 0 & 28.6802 & 28.6739 & 28.6134 & 28.3129 & 27.4558 \\
\hline & 0.1 & 35.9679 & 35.9621 & 35.889 & 35.5252 & 34.5213 \\
\hline & 0.3 & 54.0108 & 54.0021 & 53.8957 & 53.3922 & 52.0821 \\
\hline
\end{tabular}

Tables (4-6) depict the influence of rotational constant $\varphi$ on buckling load factor for different value of $\beta^{2}$. It is revealed that when $\varphi \rightarrow 0$, the boundary condition is closer to simply supported case and the loading factor shows the lower than when $\varphi \rightarrow \infty$ the outer edge is clamped and stiff against rotation.

Table 4. Buckling load factor variation with edge elastically restrained $(\beta=1)$

\begin{tabular}{|c|c|c|c|c|c|c|}
\hline \multirow{2}{*}{$\gamma$} & \multirow{4}{*}{$\varphi$} & \multicolumn{5}{|c}{$r_{b}$} \\
\cline { 3 - 7 } & & $\mathbf{0 . 1}$ & $\mathbf{0 . 2}$ & $\mathbf{0 . 3}$ & $\mathbf{0 . 4}$ & $\mathbf{0 . 5}$ \\
\hline \multirow{3}{*}{$\mathbf{0 . 3}$} & 0 & 2.39267 & 2.08653 & 1.74474 & 1.45248 & 1.22165 \\
\cline { 2 - 7 } & 10 & 7.02073 & 6.47188 & 6.4328 & 7.14899 & 8.92773 \\
\cline { 2 - 7 } & $\infty$ & 7.78485 & 7.17253 & 7.20876 & 8.21381 & 10.6283 \\
\hline \multirow{3}{*}{$\mathbf{0 . 1}$} & 0 & 3.4185 & 3.00976 & 2.59598 & 2.26171 & 2.00357 \\
\cline { 2 - 7 } & 10 & 10.1649 & 9.62109 & 9.92857 & 11.3968 & 14.4472 \\
\cline { 2 - 7 } & $\infty$ & 11.7928 & 11.2945 & 12.0246 & 14.5139 & 19.7524 \\
\hline \multirow{3}{*}{$\mathbf{0}$} & 0 & 4.0144 & 3.55674 & 3.11384 & 2.76572 & 2.50097 \\
\hline & 10 & 11.7538 & 11.2724 & 11.8074 & 13.6684 & 17.3273 \\
\hline \multirow{3}{*}{$\mathbf{0 . 1}$} & $\infty$ & 14.1309 & 13.7553 & 15.0361 & 18.5922 & 25.7854 \\
\hline & 0 & 4.66952 & 4.16614 & 3.6995 & 3.34309 & 3.07739 \\
\cline { 2 - 7 } & 10 & 13.3724 & 12.9663 & 13.7373 & 15.9742 & 20.1736 \\
\hline \multirow{3}{*}{$\mathbf{0 . 3}$} & $\infty$ & 16.7082 & 16.5284 & 18.5018 & 23.357 & 32.9186 \\
\hline & 0 & 6.17125 & 5.5881 & 5.0932 & 4.73918 & 4.49148 \\
\hline & 10 & 16.6434 & 16.4146 & 17.6377 & 20.5221 & 25.5586 \\
\hline & $\infty$ & 22.6 & 23.0801 & 26.9097 & 35.1488 & 50.8404 \\
\hline
\end{tabular}

Table 5. load factor variation with edge elastically restrained $(\beta=\sqrt{2})$

\begin{tabular}{|c|c|c|c|c|c|c|}
\hline \multirow[t]{2}{*}{$\gamma$} & \multirow[t]{2}{*}{$\varphi$} & \multicolumn{5}{|c|}{$r_{b}$} \\
\hline & & 0.1 & 0.2 & 0.3 & 0.4 & 0.5 \\
\hline \multirow{3}{*}{-0.3} & 0 & 3.86494 & 3.70778 & 3.37948 & 2.95904 & 2.54614 \\
\hline & 10 & 11.1494 & 10.7466 & 10.2355 & 10.2921 & 11.4907 \\
\hline & $\infty$ & 12.3941 & 11.9712 & 11.429 & 11.7022 & 13.4887 \\
\hline \multirow{3}{*}{-0.1} & 0 & 5.92078 & 5.68816 & 5.23808 & 4.71045 & 4.21952 \\
\hline & 10 & 16.3925 & 15.9406 & 15.5214 & 16.0831 & 18.3679 \\
\hline & $\infty$ & 19.6007 & 19.0235 & 18.7609 & 20.168 & 24.5558 \\
\hline \multirow{3}{*}{ o } & 0 & 7.16249 & 6.88791 & 6.37831 & 5.80537 & 5.28613 \\
\hline & 10 & 19.173 & 18.6768 & 18.3597 & 19.2084 & 22.0132 \\
\hline & $\infty$ & 23.9331 & 23.2797 & 23.2917 & 25.5881 & 31.8244 \\
\hline \multirow{3}{*}{0.1} & 0 & 8.55772 & 8.23958 & 7.67409 & 7.06221 & 6.52353 \\
\hline & 10 & 22.0216 & 21.1903 & 21.2775 & 22.4076 & 25.679 \\
\hline & $\infty$ & 28.762 & 28.062 & 28.4736 & 31.8876 & 40.388 \\
\hline \multirow{3}{*}{0.3} & 0 & 11.8246 & 11.4362 & 10.7724 & 10.1088 & 9.56313 \\
\hline & 10 & 27.8305 & 27.2711 & 27.2789 & 28.8982 & 32.897 \\
\hline & $\infty$ & 40.0193 & 39.3203 & 40.9454 & 47.3759 & 61.8092 \\
\hline
\end{tabular}

Table 6. load factor variation with edge elastically restrained

\begin{tabular}{|c|c|c|c|c|c|c|}
\multicolumn{7}{c}{$(\beta=\sqrt{5})$} \\
\hline \multirow{2}{*}{$\gamma$} & $\varphi$ & \multicolumn{5}{|c}{$r_{b}$} \\
\cline { 2 - 7 } & & $\mathbf{0 . 1}$ & $\mathbf{0 . 2}$ & $\mathbf{0 . 3}$ & $\mathbf{0 . 4}$ & $\mathbf{0 . 5}$ \\
\hline \multirow{3}{*}{$\mathbf{0 . 3}$} & 0 & 7.44981 & 7.42763 & 7.30188 & 6.95214 & 6.35201 \\
\cline { 2 - 7 } & 10 & 20.398 & 20.3231 & 19.9702 & 19.3557 & 19.2569 \\
\cline { 2 - 7 } & $\infty$ & 23.5367 & 23.4633 & 23.0736 & 23.396 & 22.5735 \\
\hline \multirow{3}{*}{$\mathbf{0 . 1}$} & 0 & 12.4127 & 12.3628 & 12.1602 & 11.6479 & 10.83 \\
\cline { 2 - 7 } & 10 & 31.3392 & 31.2815 & 30.8997 & 30.2359 & 30.5775 \\
\cline { 2 - 7 } & $\infty$ & 39.4941 & 39.3991 & 38.8456 & 38.3133 & 40.103 \\
\hline \multirow{3}{*}{$\mathbf{0}$} & 0 & 15.5204 & 15.469 & 15.2275 & 14.6276 & 13.70421 \\
\cline { 2 - 7 } & 10 & 37.136 & 37.0678 & 36.654 & 36.058 & 36.6338 \\
\cline { 2 - 7 } & $\infty$ & 49.4286 & 49.3058 & 48.672 & 48.401 & 51.4971 \\
\hline \multirow{3}{*}{$\mathbf{0 . 1}$} & 0 & 19.1036 & 19.0485 & 18.764 & 18.0745 & 17.0509 \\
\cline { 2 - 7 } & 10 & 43.1385 & 43.0614 & 42.6181 & 42.0768 & 42.8725 \\
\cline { 2 - 7 } & $\infty$ & 60.7884 & 60.6234 & 59.9327 & 60.0692 & 64.8462 \\
\hline \multirow{2}{*}{$\mathbf{0 . 3}$} & 0 & 27.8251 & 27.7559 & 27.3754 & 26.5071 & 25.3083 \\
\cline { 2 - 7 } & 10 & 55.7733 & 55.6721 & 55.182 & 54.7283 & 55.8625 \\
\cline { 2 - 7 } & $\infty$ & 88.0433 & 87.7881 & 87.0796 & 88.5781 & 98.01 \\
\hline \multirow{3}{*}{} & & & & & & \\
\hline
\end{tabular}

\section{2 circular solid plate}

Figures (2) and (3) illustrate the variation of bucking load factor respect to boundary condition case, orthotropic and geometry $\beta^{2}$ and $\gamma$.

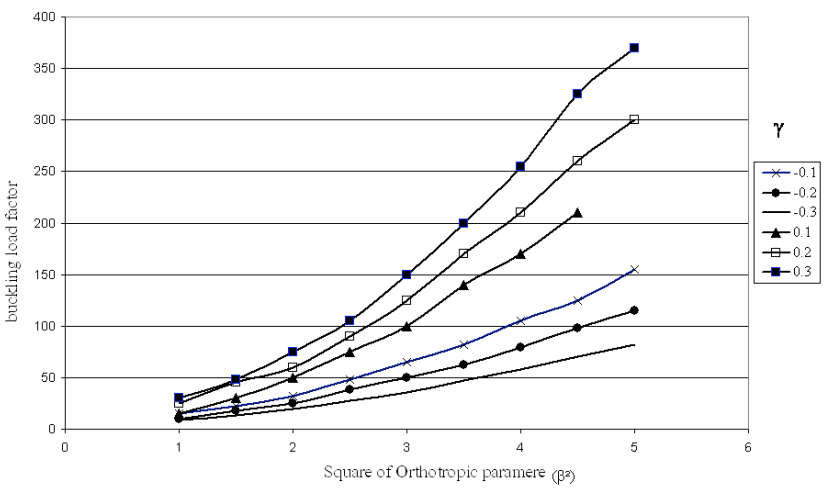

Fig. 2 variation of buckling load factor respect to orthotropic parameter and non-dimensional geometric parameter with clamped outer edge 
As shown, plate with $\gamma<0$ (centrally thicker solid plate, $\frac{h a}{h 0}<1$ ) has lower buckling load factor than plate with $\gamma>0$ (centrally thinner circular plate, $\frac{h a}{h 0}>1$ ). Buckling factor pattern for all boundary conditions are similar.

Figure (4) illustrates the influence of rotational constant $\varphi$ on buckling load factor for different value of $\beta^{2}$. For different boundary conditions, the obtained result is the same as the annular plate In fact, for simply supported outer edg, the evaluated buckling load factor is lower than clamped and elastically restrained against rotation in outer edge.

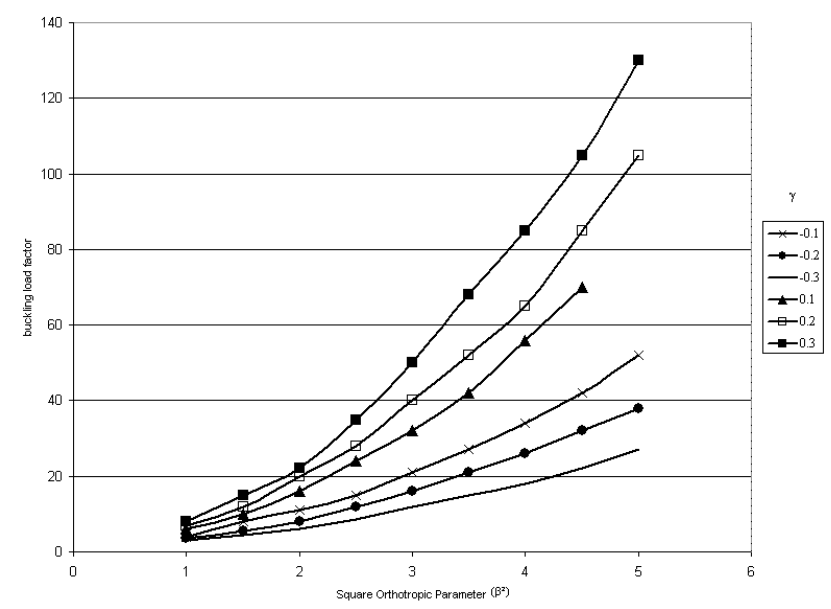

Fig. 3. Variation of buckling load factor respect to orthotropic parameter and non-dimensional geometric parameter with simply supported outer edge

As shown in Table (6), the results obtained from present method, compare very well with obtained from finite element procedure. This confirms the accuracy of Optimized Ritz method in buckling analyzing of circular plates.

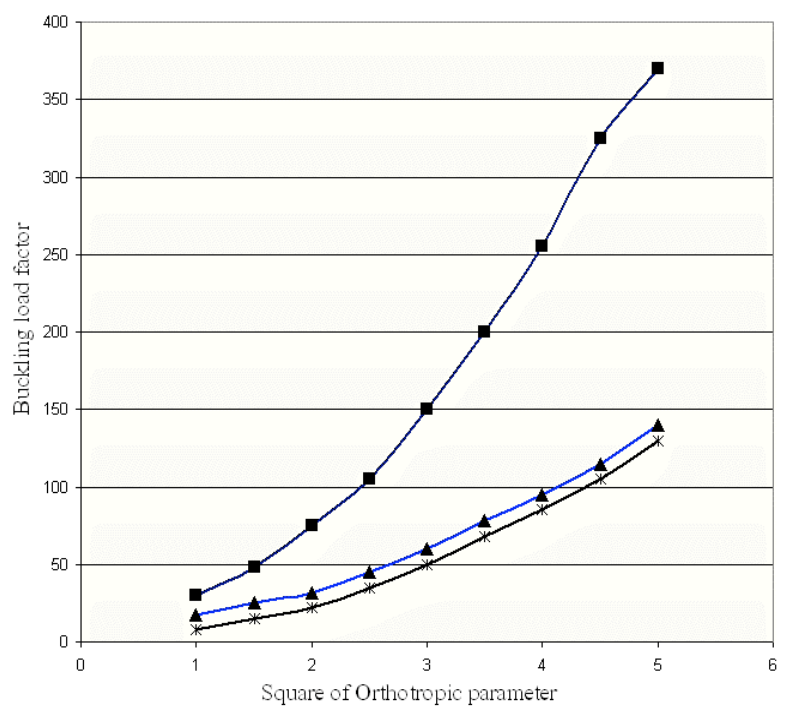

- Clamp
\#-Simply support
-Elastic

4. P.M. Ciancio, J.A. Reyes, Buckling of circular annular plates of continuously variable thickness used as internal bulkheads in submersibles, J. Ocean engineering, 30, 1323-1333, (2003).

5. B. Bremec, F. Kosel ,Thickness optimization of circular annular plates at buckling, Thin-Walled Structures, 32, 74-81, (2006).

6. R.H. Gutierrez, E. Romanlli, P.A.A Laura, Vibration and elastic stability of thin circular plates with variable profile, J. sound and vibration, 195, 391-399, (1996).

7. B. Liang, Sh. Zhang, D. Chen, Natural frequencies of circular annular plates with variable thickness by a
Fig. 4. Variation of buckling load factor respect to orthotropic parameter for different boundary conditions
Table 7. Comparison of results for buckling load factor in Optimized Ritz method (I) and FEM(II)

\begin{tabular}{|c|r|r|r|r|r|r|r|r|}
\hline \multirow{2}{*}{$\beta^{2}$} & & \multicolumn{6}{|c|}{$\gamma$} \\
\cline { 3 - 9 } & & \multicolumn{1}{|c|}{0.3} & -0.2 & -0.1 & \multicolumn{1}{c|}{0} & 0.1 & 0.2 & 0.3 \\
\hline \multirow{2}{*}{1} & I & 8.05 & 10.03 & 12.24 & 14.68 & 17.36 & 20.28 & 23.43 \\
\cline { 2 - 9 } & II & 7.8 & 9.825 & 12.11 & 14.68 & 17.53 & 20.69 & 24.15 \\
\hline \multirow{2}{*}{1.4} & I & 9.87 & 12.43 & 15.32 & 18.54 & 22.1 & 26.04 & 30.26 \\
\cline { 2 - 9 } & II & 9.72 & 12.31 & 15.24 & 18.54 & 22.21 & 26.28 & 30.75 \\
\hline \multirow{2}{*}{1.8} & I & 11.56 & 14.69 & 18.25 & 22.24 & 26.67 & 31.57 & 36.94 \\
\cline { 2 - 8 } & II & 11.55 & 14.67 & 18.23 & 22.23 & 26.7 & 31.66 & 37.11 \\
\hline
\end{tabular}

The buckling analysis of orthotropic circular annular and solid plates under uniform radial compression loading with inner edge is free while the outer edge is under different types of classical boundary conditions and also with edges elastically restrained against rotation. This is implemented by mized Ritz method. In this method, an optimization exminimized respect to it. Following are some of the concluding remarks: outer radius), increases the resistance of plate against buckling phenomena.

Plate with clamped boundary condition exhibits the higher case shows lowest. Also plate with edges elastically restrained against rotation has value of between two boundary dition cases.

eter than centrally thicker one.

\section{References}

1. S.Woinowski-Krieger, Buckling stability of circular plates with circular cylindrical Aeolotropy, IngenieurArchiv, 26, 129-13, (1958).

T. Meink, S. Huybrechts, J. Ganley, The effect of varyComposite Materials, 33, 1048-1061, (1999).

P.A.A, Laura, R.H. Gutierrez, H.C. Sanzi, G. Elvira, Buckling of circular, solid and annular plates with an intermediate circular support, J. Ocean Engineering, 27, 749-755, (2000). 
F. Farhatnia et al: Investigation on buckling of orthotropic circular and annular plates of continuously variable thickness 133

new method, J. pressure vessels and piping, 84, 293297, (2007).

8. S.P. Timoshenko, J. M. Gere, Theory of elastic stability, ( McGraw-Hill, New York, 1961).
9. U.S. Gupta, R. Lal, C.P Verm, Buckling and vibrations of polar orthotropic annular plates of variable thickness, J. sound and vibration, 104, 357-369, (1985). 